\title{
Computing Transitive Closures of Hedge Transformations
}

\author{
Tayssir Touili \\ LIAFA, CNRS \& University of Paris 7, Paris, France. \\ touili@liafa.jussieu.fr
}

\begin{abstract}
We consider the framework of regular hedge model checking where configurations are represented by trees of arbitrary arities, sets of configurations are represented by regular hedge automata, and the dynamic of a system is modeled by a term rewriting system. We consider the problem of computing the transitive closure $\mathcal{R}^{*}(L)$ of a hedge automaton $L$ and a (not necessarily structure preserving) term rewriting system $\mathcal{R}$. This construction is not possible in general. Therefore, we present a semi-algorithm that computes, in case of termination, an over-approximation of this reachability set. We show that our procedure computes the exact reachability set in many practical applications. We have successfully applied our technique to compute transitive closures for some mutual exclusion protocols defined on arbitrary width tree topologies, as well as for two interesting XML applications.
\end{abstract}

Keywords: Model-checking, Regular model-checking, Rewriting systems, Hedge automata, Transitive closures, Reachability set

\section{INTRODUCTION}

Regular Model Checking is a symbolic uniform framework for analyzing infinite-state systems in which configurations are represented by words or trees, sets of configurations by regular finite word/tree automata, and the transitions of the system by word/term rewriting systems $\left[\mathrm{KMM}^{+} 01\right.$, WB98, BJNT00, Bou01]. Then the verification problems based on performing reachability analysis of the system are reduced to the computation of closures of regular word/tree languages under word/term rewriting systems, i.e., given a regular language $L$ and a rewriting system $R$, the problem is to compute $R^{*}(L)$, where $R^{*}$ is the reflexive-transitive closure of $R$.

During the last years, several authors addressed this issue. The works done in the regular word model checking case have been successfully applied to reason about linear parametrized systems (i.e., parametrized systems where the processes are arranged in a linear topology) [BJNT00, PS00, DLS01, BMT01, Tou01, AJNd02, AJNd03], as well as systems having linear unbounded data structures such as integers, reals, lists, etc [BLW03, BHV04, BLW04], and programs with pointers [BHMV05]. As for regular tree model checking, it has been applied to the analysis of parametrized systems with tree topologies [BT02, DLS01, AJMd02, ALdR05], and multithreaded programs [LS98, BT02, BT03, BET04, Tou05].

Recently, we considered the more general case of regular hedge model checking, where configurations are represented by trees of arbitrary arities [dT06]. Indeed, arbitrary width treelike structures are very common and appear naturally in many modeling and verification contexts. For example, they appear in the following contexts:

- In the case of multithreaded recursive programs, configurations can be represented by unbounded width trees where the leaves are labeled with the control points of the program and the inner nodes with the sequential and the parallel operators - and $\|: a$ term $\|\left(t_{1}, \ldots, t_{n}\right)$ represents a configuration where the terms $t_{1}, \ldots, t_{n}$ are in parallel. In this case, we need unbounded width trees to represent these configurations since the number of parallel processes can be arbitrarily large. Using this model, actions of the program such as procedure calls, dynamic creation of new threads, synchronisation 
between parallel processes, etc, can be represented by rewriting systems on unranked trees [BT05, BMOT05, BST06].

- Several parametrized protocols are defined on unbounded width tree topologies. Indeed, in general in networks having tree topologies, the number of processes, the network topology and the arities of the different nodes are not fixed. In this case, labeled trees of arbitrary width and height are needed to represent configurations of tree networks of arbitrary numbers of processes: the skeleton of the tree represents the topology of the network, each node corresponds to one process in the network, and the label of the node represents the control state of the corresponding process. Examples of such systems are multicast protocols, leader election protocols, mutual exclusion protocols, etc.

- Unranked trees are extensively used to model XML documents [ZL03, SSM03, MBPS05]. For example, an XML document modeling a store having $n$ clients, where client $i$ bought $k_{i}$ items can be represented by a tree whose root has $n$ children, and where the $i^{t h}$ child has $k_{i}$ children. Since the number of clients and bought items are arbitrary, unranked trees are necessary to represent these systems. We can then use rewriting systems on unranked trees to represent transformations on XML documents such as XSLT (Extensible Stylesheet Language Transformation).

In [dT06], we used hedge automata [BKMW01] to symbolically represent infinite sets of unranked trees, and hedge transducers to model transformations on these trees. We proposed an acceleration technique on relabeling hedge transducers (tranducers that preserve the structure of the trees) that computes, in case of termination, the transitive closure of the hedge relabeling transformation. The semi-algorithm proposed in [dT06] works pretty well in practice. However, its main limitation is that it only deals with structure-preserving transformations, whereas in general, the transformations are not necessarily structure preserving, especially in the case of multithreaded programs and XML applications as will be seen in Section 6. In this work, we go one step further and tackle the problem of computing transitive closures for general hedge transformations, even non structure preserving ones.

The contributions of the paper can be summarized as follows:

- We formalize the concept of arbitrary hedge transformations (even non structure preserving ones) as term rewriting systems, and we give an effective construction that computes a hedge automaton representing the effect of a single application of a (linear) term rewriting system to a set of terms given by a hedge automaton. As far as we know, this is the first time that such a formalisation and such a construction are given.

- Since it is in general not possible to compute the exact reachability sets for these systems, we define a semi-algorithm that computes, in case of termination, a hedge automaton representing an over-approximation of the reachable configurations $R^{*}(L)$ for a set of terms $L$ given by a hedge automaton, and a term rewriting system $R$. Indeed, in general, it is sufficient to have an over-approximation $O$ of the reachability set since if $O$ does not contain a bad configuration, then so does the exact set of reachable configurations. We show on several examples that our procedure computes the exact reachability set in many interesting applications.

- We successfuly applied our techniques to the analysis of two mutual exclusion protocols, and two XML applications: a system that manages a store's client database, and a spam eraser. The two XML applications that we consider involve non structure preserving transformations. Hence, it is not possible to handle them with earlier work on structure preserving hedge transformations such as [dT06].

Related work. There are a lot of works that compute transitive closures for word transformations [BJNT00, DLS01, Tou01, BLW03, BHV04, BLW04, AJNd03] and tree transformations [BT02, AJMd02, ALdR05]. However, these works only consider trees where the arities are fixed, whereas our framework allows to consider ranked as well as unranked trees.

Hedge automata have been used to compute reachability sets of some classes of transformations such as Process Rewrite Systems (PRS) [BT05] and Dynamic Pushdown Networks (DPN) 
[BMOT05]. These works propose specific algorithms to compute the reachability sets for these systems. These algorithms can only be applied for the proposed model. Our algorithm is more general since it can be applied to any class of systems. It would be interesting to see if our techniques are exact for these classes for which we already have a direct construction of the reachability set.

Forest transformations have been extensively used for the analysis of XML documents. $k$-pebble tree transducers [MSV00] and macro tree transducers [MBPS05, Eng80, EV85, FV98] were used for this. The main problem of these two formalisms is that to transform forests (or equivalently, hedges), they need first to transform them into binary trees, and then perform the transformations on these binary trees. This encoding to binary trees is complex and not natural.

Recently, there has been one attempt to perform the tranformations on forests without going through binary trees in [PS04], where the notion of macro forest transducer is considered. Compared to our work, macro forest transducers define a restricted class of forest rewrite systems. Moreover, in [PS04] the authors give only an algorithm for computing immediate predecessors. They don't consider the problem of computing immediate successors, nor that of computing (over-approximations of) the reachability set.

Outline. In Section 2, we give the definition of hedge automata. Then in Section 3, we define term rewriting systems on unranked trees. In Section 4, we give our algorithm that computes the effect of the single application of a term rewriting system to a set of terms given by a hedge automaton. Section 5 describes our general semi-algorithm that computes over-approximations of the reachability sets. Finally, in Section 6 , we give some applications to which we successfully applied our technique.

\section{TERMS, HEDGES AND HEDGE AUTOMATA}

\subsection{Terms and hedges}

Let $\Sigma$ be an unranked alphabet and $\mathcal{X}$ be a fixed finite set of variables. We will use $x_{1}, x_{2}, \ldots$ to denote elements of $\mathcal{X}$. The set $T_{\Sigma}[\mathcal{X}]$ of terms over $\Sigma \cup \mathcal{X}$ is the smallest set such that:

- $\Sigma \cup \mathcal{X} \subseteq T_{\Sigma}[\mathcal{X}]$

- if $f \in \Sigma, t_{1}, \ldots, t_{n} \in T_{\Sigma}[\mathcal{X}]$ for some $n \geq 1$, then $f\left(t_{1}, \ldots, t_{n}\right) \in T_{\Sigma}[\mathcal{X}]$.

Terms without variables are called ground terms. Let $T_{\Sigma}$ be the set of ground terms over $\Sigma$. A term $t$ in $T_{\Sigma}[\mathcal{X}]$ is linear if each variable occurs at most once in $t$. $\operatorname{Var}(t)$ denotes the set of variables occuring in the term $t$. A context $C$ is a linear term of $T_{\Sigma}[\mathcal{X}]$.

As usual, a term in $T_{\Sigma}[\mathcal{X}]$ can be viewed as a rooted labeled tree where the leaves are labeled with variables or elements in $\Sigma$, and every internal node with a symbol in $\Sigma$.

A hedge is a sequence of terms. Formally, the set $H_{\Sigma}[\mathcal{X}]$ of hedges over $\Sigma \cup \mathcal{X}$ is the smallest set such that:

- $T_{\Sigma}[\mathcal{X}] \subset H_{\Sigma}[\mathcal{X}]$

- if $u \in H_{\Sigma}[\mathcal{X}]$ and $v \in H_{\Sigma}[\mathcal{X}]$, then $u v \in H_{\Sigma}[\mathcal{X}]$.

\subsection{Hedge automata}

To finitely represent infinite sets of terms, we use hedge automata [BKMW01]:

Definition 2.1 $A$ Hedge automaton is a tuple $\mathcal{A}=(Q, \Sigma, F, \delta)$ where $Q$ is a finite set of states, $\Sigma$ is an unranked alphabet, $F \subseteq Q$ is a set of final states, and $\delta$ is a set of rules of the form $f(L) \rightarrow q$, where $f \in \Sigma, q \in Q$, and $L \subseteq Q^{*}$ is a regular word language over $Q$. A rule of the form $f(\epsilon) \rightarrow q$ will simply be written $f \rightarrow q$. 
We define a move relation $\rightarrow_{\delta}$ between ground terms in $T_{\Sigma \cup Q}$ as follows: for every two terms $t$ and $t^{\prime}$, we have $t \rightarrow_{\delta} t^{\prime}$ iff there exist a context $C$ and a rule $r=f(L) \rightarrow q \in \delta$ such that $t=C\left[f\left(q_{1}, \ldots, q_{n}\right)\right], q_{1} \cdots q_{n} \in L$, and $t^{\prime}=C[q]$.

Let $\mathcal{L}$ be a finite set of regular word languages over $Q$. We extend $\rightarrow_{\delta}$ to terms over $T_{\Sigma \cup \mathcal{L}}$ as follows: $t \rightarrow \delta t^{\prime}$ iff there exist a context $C$, a rule $r=f(L) \rightarrow q \in \delta$ and $n$ regular languages from $\mathcal{L}$ such that $t=C\left[f\left(L_{1}, \ldots, L_{n}\right)\right], L_{1} \cdots L_{n} \subseteq L$, and $t^{\prime}=C[q]$, where $L_{1} \cdots L_{n}$ denotes the usual concatenation of word languages.

Let ${ }^{*} \delta$ denote the reflexive-transitive closure of $\rightarrow \delta . \stackrel{*}{\rightarrow}_{\delta}$ is extended to hedges as follows: Let $u=t_{1} \cdots t_{n}$ be a hedge. Then $u \stackrel{*}{\rightarrow} \delta q_{1} \cdots q_{n}$ iff $t_{i} \stackrel{*}{\rightarrow} \delta q_{i}$, where $q_{i} \in Q$ for $1 \leq i \leq n$. Let $u_{1}, \ldots, u_{n}$ be $n$ hedges such that for $1 \leq i \leq n, u_{i} \stackrel{*}{\rightarrow} \delta s_{i}$, where $s_{i}=q_{1}^{i} \cdots q_{k_{i}}^{i}$ is a sequence of states in $Q$. Then if $t=C\left[u_{1}, \ldots, u_{n}\right]$, we write $t=C\left[u_{1}, \ldots, u_{n}\right] \stackrel{*}{\rightarrow} \delta C\left[s_{1}, \ldots, s_{n}\right]$ instead of $C\left[q_{1}^{1}, \ldots, q_{k_{1}}^{1}, \ldots, q_{1}^{n}, \ldots, q_{k_{n}}^{n}\right]$.

A ground term $t \in T_{\Sigma}$ is accepted by a state $q \in Q$ if $t \stackrel{*}{\rightarrow}_{\delta} q$. Let $L_{q}^{\delta}=\left\{t \in T_{\Sigma} \mid t \stackrel{*}{\rightarrow}_{\delta} q\right\}$. We omit the superscript $\delta$ if it is understood from the context. A ground term $t$ is accepted (or recognized) by the automaton $\mathcal{A}$ if there is some state $q$ in $F$ such that $t \stackrel{*}{\rightarrow} \delta_{\delta} q$. The language of $\mathcal{A}$, denoted by $L(\mathcal{A})$, is the set of all ground terms accepted by $\mathcal{A}$. A set of terms $T$ over $\Sigma$ is hedge regular if there exists a hedge automaton $\mathcal{A}$ such that $T=L(\mathcal{A})$.

Intuitively, given an input term $t$, a run of $\mathcal{A}$ on $t$ according to the move relation $\rightarrow_{\delta}$ can be done in a bottom-up manner as follows: first, we assign nondeterministically a state $q$ to each leaf labeled with symbol $f$ if there is in $\delta$ a rule of the form $f(L) \rightarrow q$ s.t. $\epsilon \in L$. Then, for each node labeled with a symbol $g$, and having the terms $t_{1}, \ldots, t_{n}$ as children, we must collect the states $q_{1}, \ldots, q_{n}$ assigned to all its children, i.e., such that $t_{i} \stackrel{*}{\rightarrow} \delta q_{i}$, for $1 \leq i \leq n$, and then associate a state $q$ to the node itself if there exists in $\delta$ a rule $r=g(L) \rightarrow q$ such that $q_{1} \cdots q_{n} \in L$. A term $t$ is accepted if $\mathcal{A}$ reaches the root of $t$ in a final state.

Theorem 2.1 [BKMW01] The class of Hedge automata is effectively closed under determinization and under boolean operations. Moreover, the emptiness problem for Hedge automata is decidable.

\section{REWRITING SYSTEMS ON UNRANKED TREES}

We define in this section rewriting systems on unranked trees. Let $\mathcal{X}$ be a fixed set of variables. A substitution $\sigma$ is a mapping from $\mathcal{X}$ to $H_{\Sigma}[\mathcal{X}]$, written as $\sigma=\left\{x_{1} \rightarrow u_{1}, \ldots, x_{n} \rightarrow u_{n}\right\}$, where $u_{i}$, $1 \leq i \leq n$, is a hedge in $H_{\Sigma}[\mathcal{X}]$ that substitutes the variable $x_{i}$. The term obtained by applying the substitution $\sigma$ to a term $t$ is written $t \sigma$. We call it an instance of $t$.

Let $R$ be a rule of the form $l \rightarrow r$, where $l$ and $r$ are terms in $T_{\Sigma}[\mathcal{X}]$ such that $\operatorname{Var}(r) \subseteq \operatorname{Var}(l)$. $r$ (resp. $l$ ) is called the right hand side of $R$ (resp. the left hand side of $R$ ). $R$ is linear if $r$ is a linear term. For ground terms $t, t^{\prime}$ we write $t \rightarrow_{R} t^{\prime}$ if there exists a substitution $\sigma$ such that $t^{\prime}$ can be obtained from $t$ by replacing an occurrence of the subterm $l \sigma$ by $r \sigma . \rightarrow_{R}$ defines a relation between ground terms. Let $\rightarrow_{R}^{*}$ be the reflexive transitive closure of $\rightarrow_{R}$.

A term rewriting system (TRS) $\mathcal{R}$ is a finite set of rules of the form $l \rightarrow r$. $\mathcal{R}$ is linear if all its rules are linear. Given two ground terms $t, t^{\prime}$, we write $t \rightarrow_{\mathcal{R}} t^{\prime}$ if there exists a rule $R \in \mathcal{R}$ such that $t \rightarrow_{R} t^{\prime}$. Let $\rightarrow_{\mathcal{R}}^{*}$ be the reflexive transitive closure of $\rightarrow_{\mathcal{R}}$. Given a TRS $\mathcal{R}$ and a set of terms $T \subseteq T_{\Sigma}$, the language $\mathcal{R}(T)$ is the set of all ground terms that can be obtained from the terms in $T$ by applying once a rule from $\mathcal{R}$, i.e.,

$$
\mathcal{R}(T)=\left\{t \in T_{\Sigma} \mid \exists t^{\prime} \in T, t^{\prime} \rightarrow_{\mathcal{R}} t\right\}
$$


The language $\mathcal{R}^{*}(T)$ is defined as the set of all ground terms that can be obtained from the terms in $T$ by applying a finite number of times the rules from $\mathcal{R}$, i.e.,

$$
\mathcal{R}^{*}(T)=\left\{t \in T_{\Sigma} \mid \exists t^{\prime} \in T, t^{\prime} \rightarrow \mathcal{R}_{\mathcal{R}}^{*} t\right\}
$$

\section{COMPUTING $\mathcal{R}(T)$}

We prove in this section that for any hedge regular language $T$, and linear term rewriting system $\mathcal{R}$, the set $\mathcal{R}(T)$ is effectively hedge regular. Let $\mathcal{A}=(Q, \Sigma, F, \delta)$ be a hedge automaton that recognizes $T$. We compute in what follows a hedge automaton that recognizes $\mathcal{R}(T)$. First, we need to introduce some notations.

Let $u$ and $v$ be two words in $Q^{*}$. Let $L$ be a language in $Q^{*}$. We define the quotient $u^{-1} L v^{-1}$ as follows:

$$
u^{-1} L v^{-1}=\left\{w \in Q^{*} \mid u w v \in L\right\}
$$

Let $\mathcal{L}$ be the set of regular word languages over $Q$ that appear in the rules of $\delta$, i.e.,

$$
\mathcal{L}=\{L \mid \exists \text { a rule } f(L) \rightarrow q \in \delta\}
$$

and let $\mathcal{L}^{\prime}$ be the set of quotients of $\mathcal{L}$, i.e.,

$$
\mathcal{L}^{\prime}=\left\{u^{-1} L v^{-1} \mid u, v \in Q^{*}, L \in \mathcal{L}\right\}
$$

It is well known that $\mathcal{L}^{\prime}$ is finite since the set of quotients of a regular language is finite.

We are now ready to define our automaton that recognizes $\mathcal{R}(T)$. First, we need to define new states and rules that allow to recognize the subterms of the right hand sides of the rules of $\mathcal{R}$.

\subsection{Recognizing the subterms of the rules}

Let $\operatorname{Sub}_{r}(\mathcal{R})$ be the set of all the subterms of the right hand sides of the rules of $\mathcal{R}$. Let $Q_{\mathcal{R}}=$ $\left\{q_{t}^{L_{1}, \ldots, L_{n}}\left|t \in \operatorname{Sub}_{r}(\mathcal{R}),\right| \operatorname{Var}(t) \mid=n>0\right.$, and $\left.L_{1}, \ldots, L_{n} \in \mathcal{L}^{\prime}\right\} \cup\left\{q_{t}\left|t \in \operatorname{Sub}_{r}(\mathcal{R}),\right| \operatorname{Var}(t) \mid=\right.$ $0\}$. Let $\delta_{\mathcal{R}}$ be the following transition rules:

- if $a \in \Sigma$ is in $S u b_{r}(\mathcal{R})$, then $a \rightarrow q_{a} \in \delta_{\mathcal{R}}$,

- if $t=f\left(t_{1}, \ldots, t_{k}\right)$ is in $S_{u b}(\mathcal{R})$ such that $\operatorname{Var}(t)=\emptyset$, then $f\left(q_{t_{1}} \cdots q_{t_{k}}\right) \rightarrow q_{t}$ is in $\delta_{\mathcal{R}}$.

- if $t=f\left(r_{1}, \ldots, r_{n}\right)$ is in $\operatorname{Sub}_{r}(\mathcal{R})$ such that $\operatorname{Var}(t)=\left\{x_{1}, \ldots, x_{k}\right\}$, then for every $L_{1}, \ldots, L_{k}$ in $\mathcal{L}^{\prime}, f(L) \rightarrow q_{t}^{L_{1}, \ldots, L_{k}}$ is in $\delta_{\mathcal{R}}$, where $L=s_{1} \cdots s_{n}$ s.t. ${ }^{1}$ for $i, 1 \leq i \leq n, s_{i}=L_{j}$ if $r_{i}=x_{j}$, and if $r_{i}=t_{j}$ is not a variable, then $s_{i}=q_{t_{j}}^{L_{j_{1}}, \ldots, L_{j_{m}}}$ if $\operatorname{Var}\left(t_{j}\right)=\left\{x_{j_{1}}, \ldots, x_{j_{m}}\right\} \subseteq \operatorname{Var}(t)$, and $s_{i}=q_{t_{j}}$ if $\operatorname{Var}\left(t_{j}\right)=\emptyset$.

Then, with the rules above we can show the following:

Lemma 4.1 Let $t \in S u b_{r}(\mathcal{R})$ such that $\operatorname{Var}(t)=\left\{x_{1}, \ldots, x_{n}\right\}$. Let $L_{1}, \ldots, L_{n} \in \mathcal{L}^{\prime}$ and let $s_{1} \in L_{1}, \ldots, s_{n} \in L_{n}$. Then $t\left[s_{1}, \ldots, s_{n}\right] \stackrel{*}{\rightarrow} \delta_{\mathcal{R}} q_{t}^{L_{1}, \ldots, L_{n}}$. Moreover, if $\operatorname{Var}(t)=\emptyset$, then ${ }^{*} \stackrel{*}{\rightarrow}_{\delta_{\mathcal{R}}} q_{t}$.

\subsection{An automaton for $\mathcal{R}(T)$}

We define the automaton $\mathcal{A}_{\mathcal{R}}=\left(Q^{\prime}, \Sigma, F^{\prime}, \delta^{\prime}\right)$ as follows:

- $Q^{\prime}=Q \cup Q_{\mathcal{R}} \cup\{(q, R) \mid q \in Q\}$.

- $F^{\prime}=\{(q, R) \mid q \in F\}$.

- $\delta^{\prime}$ is the smallest set of rules containing $\delta \cup \delta_{\mathcal{R}}$ and such that:

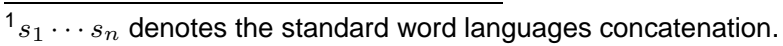


1. if $l \rightarrow r \in \mathcal{R}, \operatorname{Var}(l)=\left\{x_{1}, \ldots, x_{n}\right\}, \operatorname{Var}(r)=\left\{x_{i_{1}}, \ldots, x_{i_{k}}\right\} \subseteq \operatorname{Var}(l)$, and there exist $q \in Q$ and $L_{1}, \ldots, L_{n}$ in $\mathcal{L}^{\prime}$ such that $l\left[L_{1}, \ldots, L_{n}\right] \stackrel{*}{\rightarrow} \delta q$, then $q_{r}^{L_{i_{1}}, \ldots, L_{i_{k}}} \rightarrow(q, R)$ is in $\delta^{\prime}$.

2. if $l \rightarrow r \in \mathcal{R}, \operatorname{Var}(l)=\left\{x_{1}, \ldots, x_{n}\right\}, \operatorname{Var}(r)=\emptyset$, and there exist $q \in Q$ and $L_{1}, \ldots, L_{n}$ in $\mathcal{L}^{\prime}$ such that $l\left[L_{1}, \ldots, L_{n}\right] \stackrel{*}{\rightarrow} \delta$, then $q_{r} \rightarrow(q, R)$ is in $\delta^{\prime}$.

3. if $f(L) \rightarrow q$ is in $\delta$, then $f\left(L^{R}\right) \rightarrow(q, R)$ is in $\delta^{\prime}$, where

$$
L^{R}=\left\{q_{1} \cdots q_{i-1}\left(q_{i}, R\right) q_{i+1} \cdots q_{k} \mid q_{1} \cdots q_{i-1} q_{i} q_{i+1} \cdots q_{k} \in L\right\} .
$$

Note that the word language $L^{R}$ is regular and that we can easily compute a word automaton that accepts it if we are given a word automaton that accepts $L$.

Theorem 4.1 Let $T$ be a hedge regular set of terms, $\mathcal{A}=(Q, \Sigma, F, \delta)$ be a hedge automaton that recognizes $T$ and $\mathcal{R}$ be a linear term rewriting system. Then, $\mathcal{R}(T)$ is recognized by the hedge automaton $\mathcal{A}_{\mathcal{R}}$.

Intuition. Let us sketch the proof idea. To show that $\mathcal{R}(T)$ is accepted by $\mathcal{A}_{\mathcal{R}}$, it suffices to show that for every $q \in Q$, the state $(q, R)$ accepts the immediate successors by $\mathcal{R}$ of $L_{q}^{\delta}$ (i.e., $\left.L_{(q, R)}^{\delta^{\prime}}=\mathcal{R}\left(L_{q}^{\delta}\right)\right)$

Rules (1) express that if $l \rightarrow r$ is a rule in $\mathcal{R}$ such that $\operatorname{Var}(l)=\left\{x_{1}, \ldots, x_{n}\right\}, \operatorname{Var}(r)=$ $\left\{x_{i_{1}}, \ldots, x_{i_{k}}\right\} \subseteq \operatorname{Var}(l)$, and if $L_{1}, \ldots, L_{n}$ are $n$ regular word languages in $\mathcal{L}^{\prime}$ such that $l\left[L_{1}, \ldots, L_{n}\right] \stackrel{*}{\rightarrow} \delta q$. Then, there exist $n$ hedges $u_{1}, \cdots, u_{n} \in H_{\Sigma}$ such that for every $i$, $1 \leq i \leq n, u_{i} \stackrel{*}{\rightarrow} \delta s_{i}, s_{i} \in Q^{*}, s_{i} \in L_{i}$, and $l\left[u_{1}, \ldots, u_{n}\right] \stackrel{*}{\rightarrow}_{\delta} q$. Since $l\left[u_{1}, \ldots, u_{n}\right] \rightarrow \mathcal{R}$ $r\left[u_{i_{1}}, \ldots, u_{i_{k}}\right], r\left[u_{i_{1}}, \ldots, u_{i_{k}}\right]$ is a successor of $L_{q}$. Therefore, $r\left[u_{i_{1}}, \ldots, u_{i_{k}}\right]$ should be accepted by the state $(q, R)$. This is ensured by rules (1) together with the rules $\delta_{\mathcal{R}}$. Indeed, we have that $r\left[u_{i_{1}}, \ldots, u_{i_{k}}\right] \stackrel{*}{\rightarrow} \delta\left[s_{i_{1}}, \ldots, s_{i_{k}}\right]$ since for $1 \leq i \leq n, u_{i} \stackrel{*}{\rightarrow} \delta s_{i}$. From Lemma 4.1, we get that $r\left[s_{i_{1}}, \ldots, s_{i_{k}}\right] \stackrel{*}{\rightarrow} \delta_{\mathcal{R}} q_{r}^{L_{i_{1}}, \ldots, L_{i_{k}}}$. Hence, we obtain that

$$
r\left[u_{i_{1}}, \ldots, u_{i_{k}}\right] \stackrel{*}{\rightarrow} \delta^{\prime} q_{r}^{L_{i_{1}}, \ldots, L_{i_{k}}} \rightarrow_{\delta^{\prime}}(q, R)
$$

where the last derivation is due to Rules (1).

Note that here, it suffices to consider word languages in $\mathcal{L}^{\prime}$. Indeed, the derivation $l\left[L_{1}, \ldots, L_{n}\right] \stackrel{*}{\rightarrow}_{\delta}$ $q$ uses rules of $\delta$ of the form $f(L) \rightarrow q^{\prime}$. Therefore, to be able to perform such a derivation, the languages $L_{1}, \ldots, L_{n}$ need to be quotients of languages that appear in rules of the form $f(L) \rightarrow q^{\prime}$ in $\delta$, i.e., these languages need to be in $\mathcal{L}^{\prime}$.

Rules (2) deal with the special case where $\operatorname{Var}(r)=\emptyset$.

Rules (3) express that if $f \in \Sigma, t_{1}, \ldots, t_{n}$ are ground terms in $T_{\Sigma}$, and $q_{1}, \ldots, q_{n}, q$ are states in $Q$ such that $f\left(t_{1}, \ldots, t_{n}\right) \stackrel{*}{\rightarrow} \delta f\left(q_{1}, \ldots, q_{n}\right) \rightarrow_{\delta} q$, and if $t_{i} \rightarrow_{\mathcal{R}} t_{i}^{\prime}$ for some $i, 1 \leq i \leq n$. Then $t_{i}^{\prime}$ is accepted by the state $\left(q_{i}, R\right)$. Moreover, we have $f\left(t_{1}, \ldots, t_{n}\right) \rightarrow_{\mathcal{R}} f\left(t_{1}, \ldots, t_{i-1}, t_{i}^{\prime}, t_{i+1}, \ldots, t_{n}\right)$. Therefore, $f\left(t_{1}, \ldots, t_{i-1}, t_{i}^{\prime}, t_{i+1}, \ldots, t_{n}\right)$ should be accepted by $(q, R)$. This is ensured by Rules (3) since they allow the following derivation:

$$
f\left(t_{1}, \ldots, t_{i-1}, t_{i}^{\prime}, t_{i+1}, \ldots, t_{n}\right) \stackrel{*}{\rightarrow} \delta^{\prime} f\left(q_{1}, \ldots, q_{i-1},\left(q_{i}, R\right), q_{i+1}, \ldots, q_{n}\right) \rightarrow_{\delta^{\prime}}(q, R)
$$

The importance of linearity. Let us explain why do we need the rewriting system $\mathcal{R}$ to be linear. We show this on an example. Let $\mathcal{R}$ be the non linear rule $a(x) \rightarrow f(x, x)$. Let $u$ be a hedge accepted in $\delta$ by the sequence of states $s$, i.e. $u \stackrel{*}{\rightarrow}_{\delta} s$. Suppose that the term $a(u)$ is accepted by the state $q$, i.e., $a(u) \stackrel{*}{\rightarrow} \delta a(s) \rightarrow_{\delta} q$. Since $a(u) \rightarrow_{\mathcal{R}} f(u, u)$, if we want to apply the construction 
above in this case, $f(u, u)$ should be accepted by the state $(q, R)$. To do so, the rules above ensure the derivation $f(u, u) \stackrel{*}{\rightarrow} \delta f(s, s) \rightarrow \delta^{\prime}(q, R)$. However, by allowing the derivation $f(s, s) \rightarrow_{\delta^{\prime}}(q, R)$, we can accept any term of the form $f(u, v)$ s.t. $v \stackrel{*}{\rightarrow}_{\delta} s$ even if $u \neq v$, i.e., even if $f(u, v)$ is not a successor of $a(u)$. This is why we need the rewriting system to be linear.

\subsection{A first example}

Let $\Sigma=\{f, g, a, b\}$, let $\mathcal{R}$ be the rewriting rule $\mathcal{R}=a \rightarrow f(a, b)$, and let $T$ be a hedge regular set recognized by the hedge automaton $\mathcal{A}=(Q, \Sigma, F, \delta)$, where $Q=F=\{q\}$ and $\delta$ contains the rules $a \rightarrow q$ and $g\left(q^{*}\right) \rightarrow q$. It is easy to see that $T$ is the set of terms where the leaves are labeled with $a$ and the inner nodes with $g$. Therefore, $\mathcal{R}(T)$ is the set of terms where the inner nodes are labeled with $g$, and where all the leaves are labeled with $a$ except one position that contains the term $f(a, b)$. This set is recognized by the following automaton $\mathcal{A}_{\mathcal{R}}=\left(Q^{\prime}, \Sigma, F^{\prime}, \delta^{\prime}\right)$ produced by the construction above:

- $Q^{\prime}=\left\{q,(q, R), q_{a}, q_{b}, q_{f(a, b)}\right\}$,

- $F^{\prime}=\{(q, R)\}$,

- $\delta^{\prime}$ is the following set of rules:

- $a \rightarrow q$ and $g\left(q^{*}\right) \rightarrow q$ (the initial rules of $\delta$ );

- $a \rightarrow q_{a}, b \rightarrow q_{b}, f\left(q_{a} q_{b}\right) \rightarrow q_{f(a, b)}$ (these are the rules of $\delta_{\mathcal{R}}$ );

- $q_{f(a, b)} \rightarrow(q, R)$ (this rule is generated by the Rules (2) since $a \rightarrow_{\delta} q$ );

- $g\left(q^{*}(q, R) q^{*}\right) \rightarrow(q, R)$ (this rule is generated by the Rules (3)).

\subsection{A second example}

Let us now consider an example where the rewriting rules are not ground, i.e., they involve variables. Let $\Sigma=\{f, g, a\}$. Consider the previous set of terms $T$ recognized by $\mathcal{A}=(Q, \Sigma, F, \delta)$, where $Q=F=\{q\}$ and $\delta=\left\{a \rightarrow q, g\left(q^{*}\right) \rightarrow q\right\}$. Here $\mathcal{L}^{\prime}$ contains the unique language $q^{*}$. Consider the following rewriting rule: $\mathcal{R}=g(a, x, a) \rightarrow f(x, a) . \mathcal{R}(T)$ is recognized by the following automaton $\mathcal{A}_{\mathcal{R}}=\left(Q^{\prime}, \Sigma, F^{\prime}, \delta^{\prime}\right)$ :

- $Q^{\prime}=\left\{q,(q, R), q_{a}, q_{f(x, a)}^{q^{*}}\right\}$,

- $F^{\prime}=\{(q, R)\}$,

- $\delta^{\prime}$ is the following set of rules:

- $a \rightarrow q$ and $g\left(q^{*}\right) \rightarrow q$ (the initial rules of $\delta$ );

- $a \rightarrow q_{a}, f\left(q^{*} q_{a}\right) \rightarrow q_{f(x, a)}^{q^{*}}$ (these are the rules of $\left.\delta_{\mathcal{R}}\right)$;

- $q_{f(x, a)}^{q^{*}} \rightarrow(q, R)$ (this rule is generated by the Rules (1) since $\left.g\left(a, q^{*}, a\right) \stackrel{*}{\rightarrow} \delta q\right)$;

- $g\left(q^{*}(q, R) q^{*}\right) \rightarrow(q, R)$ (this rule is generated by the Rules (3)).

\section{COMPUTING TRANSITIVE CLOSURES}

Given a hedge regular language $T$ and a term rewriting system $\mathcal{R}$, our aim in this section is to compute $\mathcal{R}^{*}(T)$. Unfortunately, this is not possible in general since the transitive closures are not necessarily hedge regular. Therefore, we propose in what follows a semi-algorithm that, in case of termination, computes an over-approximation of the reachability set $\mathcal{R}^{*}(T)$. Computing over-approximations is sufficient in many cases. Indeed, to model check a safety property e.g., it can sometimes be sufficient to compute an over-approximation of the reachability set; since if no bad configuration is present in this over-approximation, then we can be sure that the exact reachability set is also safe (does not contain any bad configuration). Of course, if the computed over-approximation contains a bad configuration, no conclusion can be made as whether this bad configuration is reachable or not. 
Let us now describe our technique. Since $\mathcal{R}^{*}(T)=\bigcup_{i \geq 0} \mathcal{R}^{i}(T)$, a naive approach to compute $\mathcal{R}^{*}(T)$ consists in computing iteratively $\mathcal{R}(T), \mathcal{R}^{2}(T), \mathcal{R}^{3}(T), \ldots$ etc, until we find an index $k$ for which $\mathcal{R}^{k+1}(T) \subseteq \bigcup_{i \leq k} \mathcal{R}^{i}(T)$. However, as explained previously, this technique will never terminate in all non trivial cases.

Example. To illustrate this termination problem, let us come back to the previous example described in Section 4.3 where $\mathcal{R}=a \rightarrow f(a, b)$ and $T$ is the set of terms where the leaves are labeled with $a$ and the inner nodes with $g$. Then it is easy to see that a naive application of the iterative procedure described above will never terminate. Indeed $\mathcal{R}(T)$ is the set of terms where the inner nodes are labeled with $g$, and where all the leaves are labeled with $a$ except one position that contains the term $f(a, b) . \mathcal{R}^{2}(T)$ is the set of terms where the inner nodes are labeled with $g$, and where all the leaves are labeled with $a$ except one position that contains the term $f(f(a, b), b)$ union the set of terms where the inner nodes are labeled with $g$, and where all the leaves are labeled with $a$ except two positions that contain the term $f(a, b) . \mathcal{R}^{3}(T)$ is the set of terms where the inner nodes are labeled with $g$, and where all the leaves are labeled with $a$ except one position that contains the term $f(f(f(a, b), b), b)$ union the set of terms where the inner nodes are labeled with $g$, and where all the leaves are labeled with $a$ except two positions where one contains the term $f(a, b)$ and the other the term $f(f(a, b), b)$, union the set of terms where the inner nodes are labeled with $g$, and where all the leaves are labeled with $a$ except three positions that contain the term $f(a, b)$. You can go on for ever and compute $\mathcal{R}^{i}(T)$, the computation will never terminate.

To overcome this termination problem, we will compute an over-approximation of $\mathcal{R}^{*}(T)$ by applying the iterative procedure above, while adding at each step an over-approximation of the effect of a single application of $\mathcal{R}$, i.e., an over-approximation of $\mathcal{R}(T)$. We terminate if the computed set $S$ is such that $\mathcal{R}(S) \subseteq S$. This test can be done since we can compute $\mathcal{R}(S)$ and since inclusion is decidable for hedge automata (Theorem 2.1). In case of termination, our procedure is guaranteed to produce an over-approximation of the reachability set. As we will see in several examples, our procedure is exact (computes the exact reachability set) in many interesting cases.

\subsection{Computing an over-approximation of $\mathcal{R}(T)$}

We give in this section a construction that computes an over-approximation of $\mathcal{R}(T)$ for a hedge regular set of terms $T$ and a rewrite system $\mathcal{R}$. Let $\mathcal{A}=(Q, \Sigma, F, \delta)$ be a hedge automaton that recognizes $T$. The intuitive idea is to apply the previous construction (the one that computes the exact set $\mathcal{R}(T)$ ) while merging the states $(q, R)$ and $q$. Indeed, in the previous construction, the states $(q, R)$ are meant to recognize terms obtained after one application of a rule in $\mathcal{R}$ to a term in $L_{q}^{\delta}$. The rules $\delta^{\prime}$ made sure that along a derivation of a term, only one branch can be annotated by a state of the form $(q, R)$, whereas the other branches are annotated with states $q \in Q$ (this was the role of Rules (3)). This is to ensure that $\delta^{\prime}$ accepts terms where only one rewriting occurs (the rewriting occured in the branch annotated with states $(q, R)$, whereas in the other branches annotated with states $q$, no rewriting took place). If we merge the states $(q, R)$ and $q$, we cannot ensure that only one rewriting occured. Hence, the obtained automaton recognizes terms where several rewritings took place, and we obtain therefore an over-approximation of $\mathcal{R}(T)$.

An automaton for an over-approximation of $\mathcal{R}(T)$. We define the automaton $\mathcal{A}_{\mathcal{R}}^{\prime}=$ $\left(Q^{\prime \prime}, \Sigma, F^{\prime \prime}, \delta^{\prime \prime}\right)$ as follows:

- $Q^{\prime \prime}=Q \cup Q_{\mathcal{R}}$,

- $F^{\prime \prime}=F$,

- $\delta^{\prime \prime}$ is the smallest set of rules containing $\delta \cup \delta_{\mathcal{R}}$ and such that: 
$a$. if $l \rightarrow r \in R, \operatorname{Var}(l)=\left\{x_{1}, \ldots, x_{n}\right\}, \operatorname{Var}(r)=\left\{x_{i_{1}}, \ldots, x_{i_{k}}\right\} \subseteq \operatorname{Var}(l)$, and there exist $q \in Q$ and $L_{1}, \ldots, L_{n}$ in $\mathcal{L}^{\prime}$ such that $l\left[L_{1}, \ldots, L_{n}\right] \stackrel{*}{\rightarrow} \delta$, then $q_{r}^{L_{i_{1}}, \ldots, L_{i_{k}}} \rightarrow q$ is in $\delta^{\prime \prime}$.

b. if $l \rightarrow r \in R, \operatorname{Var}(l)=\left\{x_{1}, \ldots, x_{n}\right\}, \operatorname{Var}(r)=\emptyset$, and there exist $q \in Q$ and $L_{1}, \ldots, L_{n}$ in $\mathcal{L}^{\prime}$ such that $l\left[L_{1}, \ldots, L_{n}\right] \stackrel{*}{\rightarrow} \delta q$, then $q_{r} \rightarrow q$ is in $\delta^{\prime \prime}$.

Theorem 5.1 Let $T$ be a hedge regular set of terms, $\mathcal{A}=(Q, \Sigma, F, \delta)$ be a hedge automaton that recognizes $T$, and $\mathcal{R}$ be a linear rewriting system. Then, the hedge automaton $\mathcal{A}_{\mathcal{R}}^{\prime}$ recognizes an over-approximation of $\mathcal{R}(T)$.

Example. Let us come back to the example described in Section 4.3 and see how our algorithm produces the exact reachability set. The first step of our iterative procedure gives the automaton $\mathcal{A}_{\mathcal{R}}^{\prime}=\left(Q^{\prime \prime}, \Sigma, F^{\prime \prime}, \delta^{\prime \prime}\right)$ produced by the construction above:

- $Q^{\prime \prime}=\left\{q, q_{a}, q_{b}, q_{f(a, b)}\right\}$,

- $F^{\prime \prime}=\{q\}$,

- $\delta^{\prime \prime}$ is the following set of rules:

- $a \rightarrow q$ and $g\left(q^{*}\right) \rightarrow q$ (the initial rules of $\delta$ );

- $a \rightarrow q_{a}, b \rightarrow q_{b}, f\left(q_{a}, q_{b}\right) \rightarrow q_{f(a, b)}$ (these are the rules of $\left.\delta_{\mathcal{R}}\right)$;

- $q_{f(a, b)} \rightarrow q$ (this rule is generated by the Rules $(b)$ since $a \rightarrow_{\delta} q$ ).

Since this automaton does not recognize all the reachable terms, we need to apply our construction once more (this time, starting from $\mathcal{A}_{\mathcal{R}}^{\prime}$ ). We obtain the following automaton $\mathcal{A}_{\mathcal{R}}^{\prime \prime}=$ $\left(Q^{\prime \prime}, \Sigma, F^{\prime \prime}, \delta^{\prime \prime \prime}\right)$, where : $\delta^{\prime \prime \prime}$ is the following set of rules:

- $a \rightarrow q$ and $g\left(q^{*}\right) \rightarrow q, a \rightarrow q_{a}, b \rightarrow q_{b}, f\left(q_{a}, q_{b}\right) \rightarrow q_{f(a, b)}, q_{f(a, b)} \rightarrow q$ (the initial rules of $\left.\delta^{\prime \prime}\right)$;

- $q_{f(a, b)} \rightarrow q_{a}$ (this rule is generated by the Rules $(b)$ since $a \rightarrow_{\delta^{\prime \prime}} q_{a}$ ).

It is easy to verify that this automaton recognizes the exact reachability set $\mathcal{R}^{*}(T)$.

\subsection{Computing the exact reachability set}

As shown in the previous example, our techniques can compute the exact reachability set $\mathcal{R}^{*}(T)$. We show in what follows how to check whether the computed set is exact or not. First, let us introduce a notation:

Definition 5.1 A term rewrite system $\mathcal{R}$ is well-founded if there is no infinite sequence of terms $t_{0}, t_{1}, \ldots$ such that for every $i \geq 0, t_{i+1} \rightarrow \mathcal{R} t_{i}$.

Proposition 5.1 [FO97] If $\mathcal{R}$ is well-founded then

$$
T^{\prime}=\mathcal{R}^{*}(T) \text { iff } T^{\prime}=\mathcal{R}\left(T^{\prime}\right) \cup T .
$$

Thus, if $\mathcal{R}$ is well-founded, we can use our techniques to compute (an over-approximation of) the reachability set and apply then the test given above to check whether the computed set is exact or not. Notice that this test can be performed since hedge automata are closed under union and since inclusion is decidable for them (Theorem 2.1).

\section{APPLICATIONS}

In this section, we give the results of applying our techniques to the analysis of two mutual exclusion protocols defined on arbitrary width tree-like networks, and of two XML applications. 
The rewriting rules used to model the XML applications are not structure preserving. Hence, we needed the full power of our techniques to be able to handle them. Note that it is not possible to handle these XML applications with earlier work on structure preserving hedge transformations such as [dT06].

\subsection{The Unranked Simple Token Protocol}

We consider the example of the unranked simple token protocol, which is a mutual exclusion protocol defined on arbitrary width tree-like networks. Each process stores a single bit which reflects whether the process has a token or not. The process that has the token has the right to enter the critical section. In this system, the token can move from a leaf upward to the root in the following fashion: any process that currently has the token can release it to its parent. Initially, the system contains exactly one token, located in the leaves. We would like to show that the system is correct, i.e., that all the reachable configurations contain exactly one token. Moreover, we want to check that the root will receive the token, i.e., we want to check whether the configuration where the root has the token is reachable. To do so, we need to compute the set of reachable configurations. We show in what follows how our techniques allow to compute exactly this set.

Formally, let $\Sigma=\{n, \tau\}$ where $\tau$ represents a state having the token and $n$ a state without the token. The passing of the token upward the tree can be represented by the following rewriting rule: $\mathcal{R}=n(x, \tau(y), z) \rightarrow \tau(x, n(y), z)$. The initial configurations $T$ are represented by the hedge automaton $\mathcal{A}=(Q, \Sigma, F, \delta)$, where $Q=\left\{q_{0}, q_{1}\right\}, F=\left\{q_{1}\right\}$, and $\delta$ contains the rules $\tau \rightarrow q_{1}$, $n\left(q_{0}^{*}\right) \rightarrow q_{0}$ and $n\left(q_{0}^{*} q_{1} q_{0}^{*}\right) \rightarrow q_{1}$. $\mathcal{L}^{\prime}$ contains the languages $q_{0}^{*} q_{1} q_{0}^{*}$ and $q_{0}^{*}$. Then after one iteration, our algorithm produces the following hedge automaton: $\mathcal{A}_{\mathcal{R}}^{\prime}=\left(Q^{\prime \prime}, \Sigma, F^{\prime \prime}, \delta^{\prime \prime}\right)$ such that:

- $Q^{\prime \prime}=\left\{q_{0}, q_{1}, q_{n}, q_{\tau}\right\} \cup\left\{q_{\tau(x, n(y), z)}^{L_{1}, L_{2}, L_{3}} \mid L_{1}, L_{2}, L_{3} \in \mathcal{L}^{\prime}\right\}$,

- $F^{\prime \prime}=\left\{q_{1}\right\}$,

- $\delta^{\prime \prime}$ is the following set of rules:

- $\tau \rightarrow q_{1}, n\left(q_{0}^{*}\right) \rightarrow q_{0}$ and $n\left(q_{0}^{*} q_{1} q_{0}^{*}\right) \rightarrow q_{1}$ (the initial rules of $\delta$ );

- $n \rightarrow q_{n}, \tau \rightarrow q_{\tau}, n(L) \rightarrow q_{n}^{L}$ for $L \in \mathcal{L}^{\prime}$, and $\tau\left(L_{1} q_{n}^{L_{2}} L_{3}\right) \rightarrow q_{\tau(x, n(y), z)}^{L_{1}, L_{2}, L_{3}}$ s.t.

$L_{1}, L_{2}, L_{3} \in \mathcal{L}^{\prime}$ (these are the rules of $\delta_{\mathcal{R}}$ );

- $q_{\tau(x, n(y), z)}^{q_{0}^{*}, q_{0}^{*}, q_{0}^{*}} \rightarrow q_{1}$ (this rule is generated by the Rules (a) since $\left.n\left(q_{0}^{*}, \tau\left(q_{0}^{*}\right), q_{0}^{*}\right) \stackrel{*}{\rightarrow} \delta q_{1}\right)$.

This automaton represents exactly the whole set of reachable configurations, so our iterative procedure terminates with the exact reachability set.

\subsection{The Unranked Two-Way Token Protocol}

This mutual exclusion protocol is similar to the Simple Token Protocol above, with the following difference: the node that currently owns the token can release it to its parent or to one of its children. Thus, the token can move upward, as well as downward inside the tree of processes. Formally, these transformations can be represented by the following term rewriting system: $\mathcal{R}=\{n(x, \tau(y), z) \rightarrow \tau(x, n(y), z), \tau(x, n(y), z) \rightarrow n(x, \tau(y), z)\}$. As previously, starting from the initial configurations $T$ where the token is in the leaves, we would like to compute the reachability set of the system. Our techniques allow to compute exactly this set. We don't give the construction since it is similar to the previous one.

\subsection{A first $X M L$ application: A store database}

Figure 1 represents an XML document that stores the informations about the clients of a store and the items they bought. Each client has four fields: name, address, the different items that were bought, and the status of the order, i.e., whether the order is treated or not. status is 1 if the order is being treated, 0 if it has not been treated yet, and 2 if its treatment is finished. Initially, the 
first client has status 1 , and the others 0 . This document can be represented by the tree of Figure 2. Note that we need here arbitrary-width trees since the number of clients and the number of bought items are arbitrary.

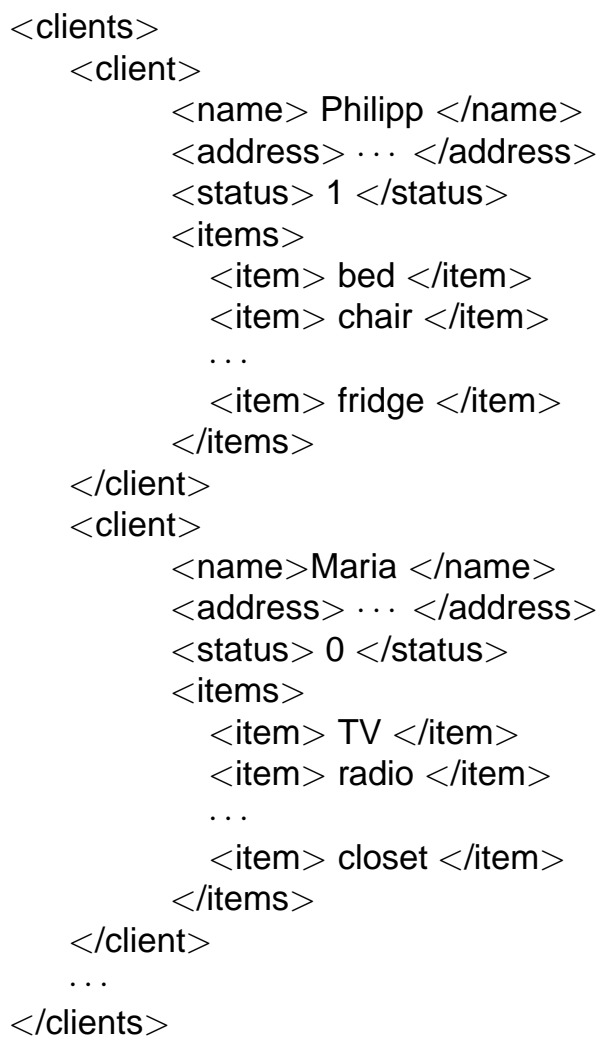

FIGURE 1: Part of a document containing information about the clients of a store

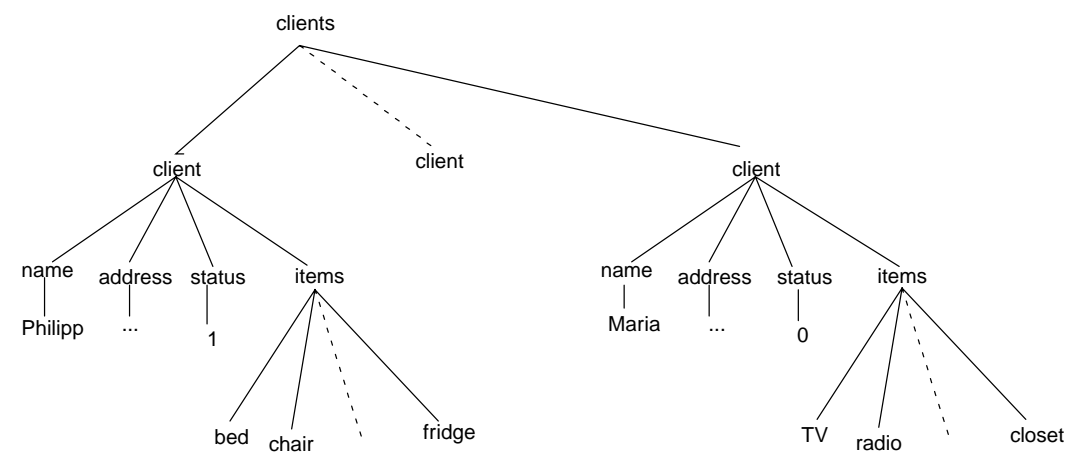

FIGURE 2: The previous XML document as a tree

The store has a software that treats the clients in the order they appear in the XML document. The software is able to:

1. Introduce a new client to the database with his name, address and the items he bought.

2. Change the status of the current client (resp. the next one) to 2 (resp. to 1) to express that the treatment of the current client is over, and that now we moved to the treatment of the next client. 
These actions can be expressed by the follwing rewrite rules. Suppose we would like to introduce into the data base a new client whose name is Eric, and who has bought a TV. Then, we need to introduce this client in the end of the queue since he has to be the last one to be treated. This is expressed by the following rewrite rule:

$$
\operatorname{clients}(x) \rightarrow \operatorname{clients}(x, \operatorname{client}(\text { name (Eric), address(street), status }(0), \text { items }(T V)))
$$

Notice that this rewrite rule is not structure preserving.

Changing the status of the current client and its neighbor can be represented by the following rewriting rule: $\operatorname{clients}\left(x_{1}, \operatorname{client}\left(x_{2}, \operatorname{status}(1), x_{3}\right), \operatorname{client}\left(x_{4}, \operatorname{status}(0), x_{5}\right), x_{6}\right) \rightarrow$ clients $\left(x_{1}, \operatorname{client}\left(x_{2}, \operatorname{status}(2), x_{3}\right), \operatorname{client}\left(x_{4}, \operatorname{status}(1), x_{5}\right), x_{6}\right)$.

In order to check the correctness of the behavior of this software, we need to compute the reachability set and check that it does not contain bad configurations where a client with a status equal to 0 (resp. 1) appears before a client with a status equal to 1 or 2 (resp. 2). This set of bad configurations can be represented by a hedge automaton. We applied our techniques to compute an over-approximation of the reachability set. We were able to check that this over-approximation does not intersect the bad configurations, and hence, that the system is correct.

\subsection{Another XML application: A spam remover}

We now consider an example of an XML application given in [MBPS05] that consists in an XML representation of a mail file containing an mbox for incoming emails and a trash folder for deleted mails. The mbox contains a spam folder to store the spam emails. Figure 3 represents an XML document that describes this:

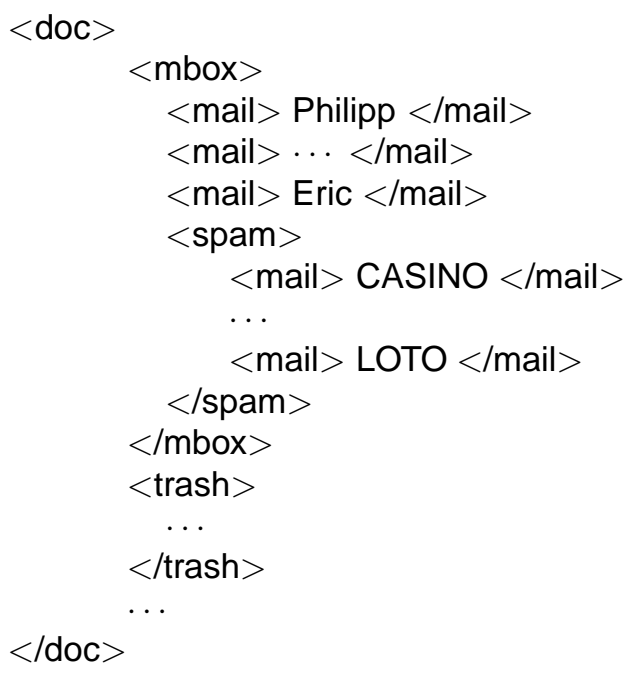

FIGURE 3: Part of a document describing a mail file

This document can be represented by the following unbounded-width term: $\operatorname{doc}(\operatorname{mbox}(\operatorname{mail}(\operatorname{Philipp}), \ldots, \operatorname{mail}(\operatorname{Eric}), \operatorname{spam}(\operatorname{mail}(C A S I N O), \operatorname{mail}(\operatorname{LOTO}), \ldots)),. \operatorname{trash}(\ldots),. \ldots .$.$) .$ Here also we need unbounded-width terms since the number of mails is unknown. This term is represented by the tree of Figure 4. 


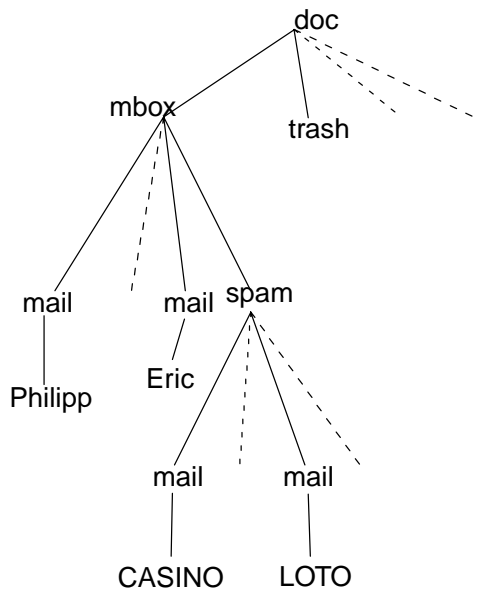

FIGURE 4: The mail document as a tree

In order to clean up the mail folder, all spam mails should be moved to the trash folder. This can be expessed by the following rewrite rule:

$$
\operatorname{doc}(\operatorname{mbox}(x, \operatorname{spam}(y)), \operatorname{trash}(z), w) \rightarrow \operatorname{doc}(\operatorname{mbox}(x, \operatorname{spam}), \operatorname{trash}(z, y), w)
$$

Note that this rule changes the structure of the terms to which it is applied.

We applied the algorithm of Section 4 and we were able to show that the system is correct after applying once this transformation, i.e., that if we start from a given set of configurations, the spam folder becomes empty after applying once the above rule.

\section{CONCLUSION}

In this paper, we considered the framework of regular hedge model checking where configurations are represented by trees of arbitrary arities, sets of configurations are represented by regular hedge automata, and the dynamic of a system is modeled by a term rewriting system. We have first formalized the concept of arbitrary hedge transformations (even non structure preserving ones) as term rewriting systems. Then, our main goal was to compute the sets $\mathcal{R}(T)$ and $\mathcal{R}^{*}(T)$, for a term rewriting system $\mathcal{R}$ and a hedge regular set of terms $T$ given by a hedge automaton. We gave an effective construction that computes a hedge automaton representing $\mathcal{R}(T)$ if $\mathcal{R}$ is a linear term rewriting system. Then, since it is in general not possible to compute the transitive closure $\mathcal{R}^{*}(T)$ for these systems, we have defined a semi-algorithm that computes, in case of termination, a hedge automaton representing an over-approximation of the reachable configurations $\mathcal{R}^{*}(T)$ for any linear term rewriting system $\mathcal{R}$. Indeed, in general, it is sufficient to have an over-approximation of the reachability set since if this over-approximation does not contain a bad configuration, then so does the exact set of reachable configurations. We showed that our procedure computes the exact reachability set in many interesting applications.

We successfuly applied our techniques to the analysis of two mutual exclusion protocols, and two XML applications: a system that manages a store's client database, and a spam eraser. These two $\mathrm{XML}$ applications involve non structure preserving transformations. Therefore, it was not possible to handle them with earlier work on structure preserving hedge transformations such as [dT06].

\section{REFERENCES}

[[AJMd02]] Parosh Aziz Abdulla, Bengt Jonsson, Pritha Mahata, and Julien d'Orso. Regular tree model checking. In Proc. $14^{\text {th }}$ Int. Conf. on Computer Aided Verification, volume 2404 of Lecture Notes in Computer Science, pages 555-568, 2002.

[[AJNd02]] Parosh Aziz Abdulla, Bengt Jonsson, Marcus Nilsson, and Julien d'Orso. Regular model checking made simple and efficient. In Proc. CONCUR 2002, $13^{\text {th }}$ Int. Conf. 
on Concurrency Theory, volume 2421 of Lecture Notes in Computer Science, pages 116-130, 2002.

[[AJNd03]] Parosh Aziz Abdulla, Bengt Jonsson, Marcus Nilsson, and Julien d'Orso. Algorithmic improvements in regular model checking. In Proc. $15^{\text {th }}$ Int. Conf. on Computer Aided Verification, volume 2725 of Lecture Notes in Computer Science, pages 236-248, 2003.

[[ALdR05]] P. A. Abdulla, A. Legay, J. d'Orso, and A. Rezine. Simulation-based iteration of tree transducers. Proceedings of TACAS'05, 2005.

[[BET04] ] A. Bouajjani, J. Esparza, and T. Touili. Reachability Analysis of Synchronised PA systems. In INFINITY'04. ENTCS, 2004.

[[BHMV05]] A. Bouajjani, P. Habermehl, P. Moro, and T. Vojnar. Verifying programs with dynamic 1-selector-linked structures in regular model checking. Proceedings of TACAS'05, 2005.

[[BHV04] ] A. Bouajjani, P. Habermehl, and T. Vojnar. Abstract regular model checking. In CAV04, Lecture Notes in Computer Science, pages 372-386, Boston, July 2004. Springer-Verlag.

[[BJNT00]] A. Bouajjani, B. Jonsson, M. Nilsson, and T. Touili. Regular model checking. In Emerson and Sistla, editors, Proc. $12^{\text {th }}$ Int. Conf. on Computer Aided Verification, volume 1855 of Lecture Notes in Computer Science, pages 403-418. Springer Verlag, 2000.

[[BKMW01]] A. Bruggemann-Klein, M. Murata, and D. Wood. Regular tree and regular hedge languages over unranked alphabets. Research report, 2001.

[[BLW03] ] Bernard Boigelot, Axel Legay, and Pierre Wolper. Iterating transducers in the large. In Proc. $15^{\text {th }}$ Int. Conf. on Computer Aided Verification, volume 2725 of Lecture Notes in Computer Science, pages 223-235, 2003.

[[BLW04] ] Bernard Boigelot, Axel Legay, and Pierre Wolper. Omega regular model checking. In Proc. TACAS '04, $10^{\text {th }}$ Int. Conf. on Tools and Algorithms for the Construction and Analysis of Systems, Lecture Notes in Computer Science, pages 561-575, 2004.

[[BMOT05]] Ahmed Bouajjani, Markus Müller-Olm, and Tayssir Touili. Regular symbolic analysis of dynamic networks of pushdown systems. In CONCUR'05, LNCS, 2005.

[[BMT01] ] A. Bouajjani, A. Muscholl, and T. Touili. Permutation rewriting and algorithmic verification. In Proc. LICS' $0117^{\text {th }}$ IEEE Int. Symp. on Logic in Computer Science. IEEE, 2001.

[[Bou01] ] A. Bouajjani. Languages, Rewriting systems, and Verification of Infinte-State Systems. In ICALP'01. LNCS 2076, 2001. invited paper.

[[BST06] ] A. Bouajjani, J. Strejček, and T. Touili. Symbolic reachability analysis of weakly extended process rewrite systems. Proc. of the 13th International Workshop on Expressiveness in Concurrency (EXPRESS'06), 2006.

[[BT02] ] Ahmed Bouajjani and Tayssir Touili. Extrapolating Tree Transformations. In Proc. $14^{\text {th }}$ Int. Conf. on Computer Aided Verification, volume 2404 of Lecture Notes in Computer Science, pages 539-554, 2002.

[[BT03] ] A. Bouajjani and T. Touili. Reachability analysis of process rewrite systems. In FSTTCS03, Lecture Notes in Computer Science, pages 73-87, 2003.

[[BT05] ] A. Bouajjani and T. Touili. On computing reachability sets of process rewrite systems. In Proc. $16^{\text {th }}$ Int. Conf. on Rewriting Techniques and Applications (RTA'05), volume 3467 of Lecture Notes in Computer Science, April 2005.

[[DLS01] ] D. Dams, Y. Lakhnech, and M. Steffen. Iterating transducers. In G. Berry, H. Comon, and A. Finkel, editors, Computer Aided Verification, volume 2102 of Lecture Notes in Computer Science, pages 286-297, 2001.

[[dT06] ] Julien d'Orso and Tayssir Touili. Regular hedge model checking. Proc. of the 4th IFIP International Conference on Theoretical Computer Science (TCS'06), 2006.

[[Eng80] ] J. Engelfriet. Some open questions and recent results on tree transducers and tree languages. Formal language theory: perspectives and open problems (R.V. Book, ed.), 1980.

[[EV85] ] J. Engelfriet and H. Vogler. Macro tree transducers. Journal of Computer and System Sciences, 1985. 
[[FO97] ] L. Fribourg and H. Olsen. Reachability sets of parametrized rings as regular languages. In Infinity'97. volume 9 of Electronical Notes in Theoretical Computer Science. Elsevier Science, 1997.

[[FV98] ] Zoltan Fulop and H. Vogler. Syntax-Directed Semantics: Formal Models Based on Tree Transducers. Springer-Verlag New York, Inc., Secaucus, NJ, USA, 1998.

[[KMM+ 01]] Y. Kesten, O. Maler, M. Marcus, A. Pnueli, and E. Shahar. Symbolic model checking with rich assertional languages. Theoretical Computer Science, 256:93-112, 2001.

[[LS98] ] D. Lugiez and Ph. Schnoebelen. The regular viewpoint on PA-processes. In Proc. 9th Int. Conf. Concurrency Theory (CONCUR'98), Nice, France, Sep. 1998, volume 1466, pages 50-66. Springer, 1998.

[[MBPS05]] S. Maneth, A. Berlea, T. Perst, and H. Seidl. Xml type checking with macro tree transducers. 24th ACM SIGACT-SIGMOD-SIGART Symp. on Principles of Database Systems (PODS), 2005.

[[MSV00] ] Tova Milo, Dan Suciu, and Victor Vianu. Typechecking for xml transformers. Symposium on Principles of Database Systems (PODS), 2000.

[[PS00] ] A. Pnueli and E. Shahar. Liveness and acceleration in parametrized verification. In CAV'00. LNCS, 2000.

[[PS04] ] Thomas Perst and Helmut Seidl. Macro forest transducers. Information Processing Letters, 2004.

[[SSM03] ] H. Seidl, Th. Schwentick, and A. Muscholl. Numerical Document Queries. In PODS'03. ACM press, 2003.

[[Tou01] ] T. Touili. Regular Model Checking using Widening Techniques. Electronic Notes in Theoretical Computer Science, 50(4), 2001. Proc. Workshop on Verification of Parametrized Systems (VEPAS'01), Crete, July, 2001.

[[Tou05] ] T. Touili. Dealing with communication for dynamic multithreaded recursive programs. In 1st VISSAS workshop, 2005. Invited Paper.

[[WB98] ] Pierre Wolper and Bernard Boigelot. Verifying systems with infinite but regular state spaces. In Proc. 10th Int. Conf. on Computer Aided Verification, volume 1427 of Lecture Notes in Computer Science, pages 88-97, Vancouver, July 1998. Springer Verlag.

[[ZL03] ] Silvano Dal Zilio and Denis Lugiez. Xml schema, tree logic and sheaves automata. In RTA'03, 2003. 